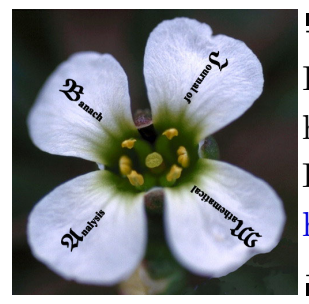

Banach J. Math. Anal. 9 (2015), no. 3, 1-13

http://doi.org/10.15352/bjma/09-3-1

ISSN: $1735-8787$ (electronic)

http://projecteuclid.org/bjma

\title{
TWO METHODS FOR THE CHARACTERIZATION OF COMPACT OPERATORS BETWEEN $B K$ SPACES
}

\author{
EBERHARD MALKOWSKY ${ }^{1}$, IVANA DJOLOVIĆ ${ }^{2 *}$ AND KATARINA PETKOVIĆ ${ }^{3}$ \\ Communicated by K. Jarosz
}

\begin{abstract}
We establish some identities or inequalities for the Hausdorff measure of noncompactness for operators $L \in \mathcal{B}(X, Y)$ when $X=\ell_{p}(1 \leq p<\infty)$ and $Y=c ; X=\ell_{p}(1<p<\infty)$ and $Y=\ell_{\infty} ; X=b v_{0}$ and $Y=c$; $X=c_{0}(\Delta), c(\Delta), \ell_{\infty}(\Delta)$ and $Y=\ell_{\infty}$. These identities and estimates are used to establish necessary and sufficient conditions for the operators to be compact. Furthermore, we apply a result by Sargent to establish necessary and sufficient conditions for operators in $\mathcal{B}\left(b v_{0}, \ell_{\infty}\right)$ and $\mathcal{B}\left(\ell_{1}, Y\right)$ to be compact, where $Y=w_{\infty}, v_{\infty},[c]_{\infty}$.
\end{abstract}

\section{INTRODUCTION}

There are two important and useful methods for the characterizations of compact linear operators between $B K$ spaces, namely the application of the Hausdorff measure of noncompactness and a result by Sargent [16].

The first method is based on the fundamental result by Goldenštein, Gohberg and Markus, for instance in [18, Theorem 4.2], and has been used in several recent papers, for instance in $[3,4,8,9,12,13,14,15]$. It can, however, only be applied when the final space has a Schauder basis. In such cases, many authors modified the proof of [10, Theorem 2.15] to obtain sufficient conditions for the compactness of operators which are not necessary, in general.

Date: Received: Jan. 13, 2014; Revised: Mar. 4, 2014.; Accepted: Apr. 1, 2014.

* Corresponding author.

2010 Mathematics Subject Classification. Primary: 46B45; Secondary: $47 \mathrm{~B} 37$.

Key words and phrases. BK spaces, bounded and compact linear operators, Hausdorff measure of noncompactness. 
One prominent case where the first method fails is the characterization of compact linear operators from $\ell_{1}$, the space of absolutely convergent series, into $\ell_{\infty}$, the space of bounded sequences. The characterization of those compact operators was given in [16, Theorem 5]. To the best of the authors' knowledge this result or a modified version have only been used in a few papers, for instance in [11, Theorem 4.8 (vi), (b)], [9, Proposition 2.5] and [3, Lemma 3.13, Corollary 3.14 7.].

We are going to both use the first method and apply [16, Theorem 5] or some modification to establish new characterizations of compact linear operators. To be able to give a more detailed survey, we need a few useful and customary notations.

A recent characterization of compact operators on $\ell_{\infty}$ can be found in $[1$, Lemma 4.1(a)].

Let $\omega$ be the set of all complex sequences $x=\left(x_{k}\right)_{k=1}^{\infty}$. By $\ell_{\infty}, c, c_{0}$ and $\phi$, we denote the sets of all bounded, convergent, null and finite sequences, respectively; we also write $\ell_{p}=\left\{x \in \omega: \sum_{k=1}^{\infty}\left|x_{k}\right|^{p}<\infty\right\}$ for $1 \leq p<\infty$, and bs and $c s$ for the sets of all bounded and convergent series.

We write $\mathcal{B}(X, Y)$ for the set of all bounded linear operators between the normed spaces $X$ and $Y$; the operator norm of $L \in \mathcal{B}(X, Y)$ is

$$
\|L\|=\sup _{\|x\|=1}\|L(x)\| .
$$

Applying the first method, we establish identities or estimates for operators $L \in \mathcal{B}(X, Y)$ when $X=\ell_{p}(1 \leq p<\infty)$ and $Y=c ; X=\ell_{p}(1<p<\infty)$ and $Y=\ell_{\infty} ; X=b v_{0}$, the intersection of $c_{0}$ and the space of all difference sequences in $\ell_{1}$ and $Y=c$; and $X=c_{0}(\Delta), c(\Delta), \ell_{\infty}(\Delta)$, the spaces of all difference sequences in $c_{0}, c$ and $\ell_{\infty}$ and $Y=\ell_{\infty}$. These identities and estimates are used to establish necessary and sufficient conditions for the operators to be compact.

The second method is applied to establish necessary and sufficient conditions for operators in $\mathcal{B}\left(b v_{0}, \ell_{\infty}\right)$ and $\mathcal{B}\left(\ell_{1}, Y\right)$ where $Y=w_{\infty}, v_{\infty},[c]_{\infty}$ to be compact, where $w_{\infty}, v_{\infty}$ and $[c]_{\infty}$ are the sets of all strongly $C_{1}$ bounded sequences, of all difference sequences in $w_{\infty}$, and of all strongly bounded sequences, respectively.

We also give simple proofs for the characterizations of the classes of matrix transformations from $X$ into $Y$, when $X=c_{0}(\Delta), c(\Delta), \ell_{\infty}(\Delta)$ and $Y=\ell_{\infty}$; and $X=\ell_{1}$ and $Y=w_{\infty}, v_{\infty},[c]_{\infty}$.

\section{Notations and Known Results}

We list the most important notations and known results which will be used throughout the paper.

Let $e$ and $e^{(n)}(n=1,2, \ldots)$ be the sequences with $e_{k}=1$ for all $k$, and $e_{n}^{(n)}=1$ and $e_{k}^{(n)}=0(k \neq n)$.

Let $X$ be any subset of $\omega$ and $z \in \omega$ be given. Then we write $z^{-1} * X=\{a \in$ $\left.\omega: a \cdot z=\left(a_{k} z_{k}\right)_{k=1}^{\infty} \in X\right\}$ and $X^{\beta}=\bigcap_{x \in X} x^{-1} * c s$ for the $\beta$-dual of $X$, that is, $a \in X^{\beta}$ if and only if $\sum_{k=1}^{\infty} a_{k} x_{k}$ converges for all $x \in X$.

A $B K$ space is a Banach sequence space $X$ with continuous coordinates $P_{n}$ $(n=1,2, \ldots)$ where $P_{n}(x)=x_{n}$ for each sequence $x=\left(x_{k}\right)_{k=1}^{\infty} \in X$; a $B K$ space 
$X \supset \phi$ is said to have $A K$ if $x^{[m]}=\sum_{k=1}^{m} x_{k} e^{(k)} \rightarrow x(m \rightarrow \infty)$ for every sequence $x=\left(x_{k}\right)_{k=1}^{\infty} \in X ; x^{[m]}$ is called the $m$-section of the sequence $x$.

Let $(X,\|\cdot\|)$ be a normed space, and $S_{X}=\{x \in X:\|x\|=1\}$ and $\overline{B_{X}}=\{x \in$ $X:\|x\| \leq 1\}$ denote the unit sphere and closed unit ball in $X$. If $(X,\|\cdot\|)$ is a normed sequence space, then we write $\|a\|_{X}^{*}=\sup _{x \in \overline{B_{X}}}\left|\sum_{k=1}^{\infty} a_{k} x_{k}\right|$ for $a \in \omega$ provided the expression on the right-hand side exists and is finite which is the case whenever $X$ is a $B K$ space and $a \in X^{\beta}$ [19, Theorem 7.2.9].

Let $A=\left(a_{n k}\right)_{n, k=1}^{\infty}$ be an infinite matrix of complex numbers, $X$ and $Y$ be subsets of $\omega$ and $x \in \omega$. We write $A_{n}=\left(a_{n k}\right)_{k=1}^{\infty}$ and $A^{k}=\left(a_{n k}\right)_{n=1}^{\infty}$ for the sequences in the $n$th row and $k$ th column of $A, A_{n} x=\sum_{k=1}^{\infty} a_{n k} x_{k}, A x=\left(A_{n} x\right)_{n=1}^{\infty}$ (provided all the series $A_{n} x$ converge), and $X_{A}=\{x \in \omega: A x \in X\}$ for the matrix domain of $A$ in $X$. Also $(X, Y)$ is the class of all matrices $A$ such that $X \subset Y_{A}$; so $A \in(X, Y)$ if and only if $A_{n} \in X^{\beta}$ for all $n$ and $A x \in Y$ for all $x \in X$.

Let $T=\left(t_{n k}\right)_{n, k=1}^{\infty}$ be a triangle, that is, $t_{n n} \neq 0$ for all $n$ and $t_{n k}=0$ for $k>n$, and $X$ be a normed sequence space with $\|\cdot\|$. Then $\|\cdot\|_{X_{T}}$ is defined by $\|x\|_{X_{T}}=\|T x\|$ for all $x \in X_{T}$; it is clear that $\|\cdot\|_{X_{T}}$ is a norm on $X_{T}$. Moreover, if $X$ is a $B K$ space with $\|\cdot\|$ then so is $X_{T}$ with $\|\cdot\|_{X_{T}}$ by [18, Theorem 4.3.12]. If $z$ is a sequence with $z_{k} \neq 0$ for all $k$, and $T$ is the diagonal matrix with $z$ on the diagonal, then $z^{-1} * X=X_{T}$ becomes a $B K$ space with $\|x\|_{z^{-1} * X}=\|z \cdot x\|$ for all $x \in X$, whenever $X$ is a $B K$ space.

The following well-known result gives the relation between $\mathcal{B}(X, Y)$ and $(X, Y)$ for $B K$ spaces $X$ and $Y$, and will frequently be used throughout.

Proposition 2.1. ([19, Theorem 4.2.8], [5, Theorem 1.9]) Let $X$ and $Y$ be $B K$ spaces.

(a) Then we have $(X, Y) \subset \mathcal{B}(X, Y)$, that is, every matrix $A \in(X, Y)$ defines an operator $L_{A} \in \mathcal{B}(X, Y)$, where $L_{A}(x)=$ Ax for all $x \in X$.

(b) If $X$ has $A K$ then we have $\mathcal{B}(X, Y) \subset(X, Y)$, that is, every operator $L \in$ $\mathcal{B}(X, Y)$ is given by a matrix $A \in(X, Y)$, where $A x=L(x)$ for all $x \in X$.

We recall that a linear operator $L$ between infinite dimensional Banach spaces $X$ and $Y$ is said to be compact if its domain is all of $X$ and, for every bounded sequence $\left(x_{n}\right)$ is $X$, the sequence $\left(L\left(x_{n}\right)\right)$ has a convergent subsequence in $Y$.

Let $(X, d)$ be a complete metric space and $\mathcal{M}_{X}$ denote the class of all bounded subsets of $X$. We write $B_{r}\left(x_{0}\right)=\left\{x \in X: d\left(x, x_{0}\right)<r\right\}$ for the open ball of radius $r>0$ and center in $x_{0} \in X$. The function $\chi: \mathcal{M}_{X} \rightarrow[0, \infty)$ with

$$
\chi(Q)=\inf \left\{\varepsilon>0: Q \subset \bigcup_{k=1}^{n} B_{r_{k}}\left(x_{k}\right), x_{k} \in X, r_{k}<\varepsilon(k=1,2, \ldots, n ; n \in \mathbb{N})\right\}
$$

is called the Hausdorff measure or ball measure of noncompactness ([18, Definition 2.1]). It has the following known properties for all $Q, Q_{1}, Q_{2} \in \mathcal{M}_{X}$ ([18, Proposition 2.3 (d), (f), (i)])

$$
\begin{aligned}
& Q_{1} \subset Q_{2} \text { implies } \chi\left(Q_{1}\right) \leq \chi\left(Q_{2}\right) \text { (monotonicity); } \\
& \chi(Q)=0 \text { for every compact set } Q ;
\end{aligned}
$$


if $X$ is a Banach space, then $\chi$ also satisfies

$$
\chi\left(Q_{1}+Q_{2}\right) \leq \chi\left(Q_{1}\right)+\chi\left(Q_{2}\right) \text { (algebraic semi-additivity). }
$$

Let $X$ and $Y$ be Banach spaces and $\chi_{1}$ and $\chi_{2}$ be Hausdorff measures of noncompactness on $X$ and $Y$. Then an operator $L: X \rightarrow Y$ is called $\left(\chi_{1}, \chi_{2}\right)_{-}$ bounded if $L(Q) \in \mathcal{M}_{Y}$ for all $Q \in \mathcal{M}_{X}$, and if there exists a constant $C>0$ such that

$$
\chi_{2}(L(Q)) \leq C \cdot \chi_{1}(Q) \text { for all } Q \in \mathcal{M}_{X} .
$$

If an operator $L$ is $\left(\chi_{1}, \chi_{2}\right)$-bounded then the number

$$
\|L\|_{\left(\chi_{1}, \chi_{2}\right)}=\inf \{C>0:(2.4) \text { holds }\}
$$

is called the $\left(\chi_{1}, \chi_{2}\right)$-measure of noncompactness of $L$.

We write $\|L\|_{\chi}=\|L\|_{\left(\chi_{1}, \chi_{2}\right)}$, for short, and call $\|L\|_{\chi}$ the Hausdorff measure of noncompactness of the operator $L$ ([10, Definition 2.24]).

It is known that if $X$ and $Y$ are infinite dimensional Banach spaces and $L \in$ $\mathcal{B}(X, Y)$, then

$$
\|L\|_{\chi}=\chi\left(L\left(\overline{B_{X}}\right)\right)=\chi\left(L\left(S_{X}\right)\right) \quad([10, \text { Theorem 2.25] })
$$

and

$L$ is compact if and only if $\|L\|_{\chi}=0 \quad([10$, Corollary $2.26(2.58)])$.

\section{Main Results}

Throughout, let $q$ denote the conjugate number of $p$ for $1 \leq p \leq \infty$, that is, $q=\infty$ for $p=1, q=p /(p-1)$ for $1<p<\infty$ and $q=1$ for $p=\infty$. We write $\|\cdot\|_{p}$ for the $B K$ norms on $\ell_{p}(1 \leq p \leq \infty)$, that is,

$$
\|x\|_{p}=\left(\sum_{k=1}^{\infty}\left|x_{k}\right|^{p}\right)^{1 / p} \text { for } 1<p<\infty \text { and }\|x\|_{\infty}=\sup _{k}\left|x_{k}\right| \text { for } p=\infty \text {. }
$$

If $A=\left(a_{n k}\right)_{n, k=1}^{\infty}$ is any infinite matrix, we write $A^{<r>}$ and $A^{>r<}$ for the matrices with the first $r$ rows and the first $r$ columns replaced by zero sequences, respectively.

Proposition 3.1. Let $1 \leq p<\infty$ and $Y=c$ or $Y=\ell_{\infty}$.

If $L \in \mathcal{B}\left(\ell_{p}, Y\right)$, then $L$ is given by an infinite matrix $A \in\left(\ell_{p}, Y\right)$ as in Proposition $2.1(b)$.

(a) If $Y=c$, then the Hausdorff measure of noncompactness of $L$ satisfies the inequalities.

$$
\frac{1}{2} \cdot \lim _{r \rightarrow \infty}\left(\sup _{n}\left\|B_{n}^{<r>}\right\|_{q}\right) \leq\|L\|_{\chi} \leq \lim _{r \rightarrow \infty}\left(\sup _{n}\left\|B_{n}^{<r>}\right\|_{q}\right),
$$

where $B$ is the matrix with the rows $B_{n}=A_{n}-\left(\alpha_{k}\right)_{k=1}^{\infty}$ for $n=1,2, \ldots$ and $\alpha_{k}=\lim _{n \rightarrow \infty} a_{n k}$ for $k=1,2, \ldots$.

(b) If $Y=\ell_{\infty}$, then the Hausdorff measure of noncompactness of $L$ satisfies the inequality

$$
0 \leq\|L\|_{\chi} \leq \lim _{r \rightarrow \infty}\left(\sup _{n}\left\|A_{n}^{<r>}\right\|_{q}\right)
$$


Proof. Since $\ell_{p}$ is a $B K$ space with $A K$ for each $p$ with $1 \leq p<\infty$, the first part is clear.

(a) If follows by [2, Theorem 3.4] and the the fact that $\ell_{p}^{*}$ and $\ell_{q}$ are norm isomorphic that

$$
\frac{1}{2} \cdot \limsup _{r \rightarrow \infty}\left(\sup _{n}\left\|B_{n}^{<r>}\right\|_{q}\right) \leq\|L\|_{\chi} \leq \limsup _{r \rightarrow \infty}\left(\sup _{n}\left\|B_{n}^{<r>}\right\|_{q}\right) .
$$

Since obviously $\left\|B_{n}^{<r>}\right\|_{q} \geq\left\|B_{n}^{<r+1>}\right\|_{q} \geq 0(r=1,2, \ldots)$ for each $n \in \mathbb{N}$, the limits exist in the last two inequalities. Hence we have established the inequalities in (3.1).

(b) Let $\mathcal{P}_{r}: \ell_{\infty} \rightarrow \ell_{\infty}$ for $r \in \mathbb{N}$ be defined by $P_{r}(x)=x^{(r)}$ for all $x \in \ell_{\infty}$ and $\mathcal{R}_{r}=I-\mathcal{P}_{r}$, where $I$ is the identity on $\ell_{\infty}$. We also write $\bar{B}=\overline{B_{\ell_{p}}}$, for short. Then it follows by (2.6), the monotonicity (2.1), algebraic semi-additivity (2.3) of $\chi$ and (2.2), and by [10, Theorem $1.23(\mathrm{~b})]$ and the fact that $\ell_{p}^{*}$ and $\ell_{q}$ are norm isomorphic

$$
\begin{aligned}
0 & \leq\|L\|_{\chi}=\chi(L(\bar{B})) \leq \chi\left(\mathcal{P}_{r}(L(\bar{B}))\right)+\chi\left(\mathcal{R}_{r}(L(\bar{B}))\right)=\chi\left(\mathcal{R}_{r}(L(\bar{B}))\right. \\
& \leq \sup _{x \in \bar{B}}\left\|\mathcal{R}_{r}(L(x))\right\|=\left\|\mathcal{R}_{r} \circ L\right\|=\left\|L_{A^{<r+1>}}\right\|=\sup _{n}\left\|A_{n}^{<r+1>}\right\|_{\ell_{p}^{*}} \\
& =\sup _{n}\left\|A_{n}^{<r+1>}\right\|_{q} \text { for all } r \in \mathbb{N} .
\end{aligned}
$$

This yields the inequalities in (3.2).

Applying (2.7) and Proposition 3.1, we obtain

Corollary 3.2. Let $1 \leq p<\infty$. We use the notations of Proposition 3.1. (a) If $L \in \mathcal{B}\left(\ell_{p}, c\right)$, then $L$ is compact if and only if

$$
\lim _{r \rightarrow \infty}\left(\sup _{n}\left\|B_{n}^{<r>}\right\|_{q}\right)=0
$$

(b) If $L \in \mathcal{B}\left(\ell_{p}, \ell_{\infty}\right)$ and

$$
\lim _{r \rightarrow \infty}\left(\sup _{n}\left\|A_{n}^{<r>}\right\|_{q}\right)=0
$$

then $L$ is compact.

We note that the condition in (3.3) is only sufficient, but not necessary, in general, for an operator $L \in \mathcal{B}\left(\ell_{p}, \ell_{\infty}\right)$ to be compact. To see this, let $L: \ell_{p} \rightarrow \ell_{\infty}$ be defined by $L(x)=x_{1} \cdot e$ for all $x \in \ell_{p}$, hence $L$ is compact. Also $L$ is given by the matrix $A$ with the rows $A_{n}=e^{(1)}$ for $n=1,2, \ldots$, but the limit in (3.3) is

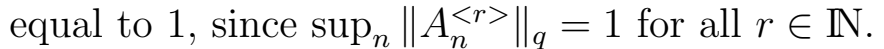

If $1<p<\infty$ the result of Corollary (3.2) (b) can be improved by applying [19, Theorem 8.3.9] and using the transpose $A^{T}$ of the matrix $A$ that represents the operator $L \in \mathcal{B}\left(\ell_{p}, \ell_{\infty}\right)$.

Proposition 3.3. Let $1<p<\infty$. If $L \in \mathcal{B}\left(\ell_{p}, \ell_{\infty}\right)$, then $L$ is compact if and only if

$$
\lim _{r \rightarrow \infty}\left(\sup _{n}\left\|A_{n}^{>r<}\right\|_{q}\right)=0 .
$$


Proof. Let $L \in \mathcal{B}\left(\ell_{p}, \ell_{\infty}\right)$ and $L$ be given by the matrix $A \in\left(\ell_{p}, \ell_{\infty}\right)$. We apply [19, Theorem 8.3.9] with $X=\ell_{p}$ and $Z=\ell_{1}, B K$ spaces with $A K, Y=Z^{\beta}=\ell_{\infty}$ and $X^{\beta}=\ell_{q}$ to obtain $A \in\left(\ell_{p}, \ell_{\infty}\right)$ if and only if $C=A^{T} \in\left(\ell_{1}, \ell_{q}\right)$; also $\|L\|=\left\|L_{C}\right\|$ by [16, Lemma 2]. Now $A \in\left(\ell_{p}, \ell_{\infty}\right)$ implies $\|L\|=\sup _{n}\left\|A_{n}\right\|_{\ell_{p}^{*}}=\sup _{n}\left\|A_{n}\right\|_{q}$ as before in the proof of Proposition 3.1 (b). It follows by (2.6), [10, Theorem 2.15 ] and since obviously $C^{<r>}=\left(A^{>r<}\right)^{T}$ that

$$
\begin{aligned}
\left\|L_{C}\right\|_{\chi} & =\chi\left(L_{C}\left(\overline{B_{\ell_{1}}}\right)\right)=\lim _{r \rightarrow \infty}\left(\sup _{x \in \overline{B_{\ell_{1}}}}\left\|\mathcal{R}_{r}\left(L_{C}(x)\right)\right\|_{q}\right)=\lim _{r \rightarrow \infty}\left\|\mathcal{R}_{r} \circ L_{C}\right\| \\
& =\lim _{r \rightarrow \infty}\left\|L_{C<r>}\right\|=\lim _{r \rightarrow \infty}\left\|L_{\left(C^{<r>}\right)^{T}}\right\|=\lim _{r \rightarrow \infty}\left\|L_{A^{>r<}}\right\| \\
& =\lim _{r \rightarrow \infty}\left(\sup _{n}\left\|A_{n}^{>r<}\right\|_{q}\right) .
\end{aligned}
$$

Since $L_{A}$ is compact if and only if $L_{C}$ is compact by [16, Theorem 3], (3.4) now follows from (2.7).

We note that the statement of Proposition 3.3 is known and can be found, for instance, in [16, (b), p. 85].

The use of the transpose of the matrix $A$ fails in the case of $p=1$, since $\ell_{1}^{\beta}=\ell_{\infty}$ and $\ell_{\infty}^{\beta}=\ell_{1}$. In this case, the characterization of compact operators is given in [16, Theorem 5]. Since we are going to use this result, we state it here for the reader's convenience in a slightly modified version.

Lemma 3.4. ([16, Theorem 5]) If $L \in \mathcal{B}\left(\ell_{1}, \ell_{\infty}\right)$, then $L$ is compact if and only if

$$
\begin{aligned}
\lim _{m \rightarrow \infty} \sup _{1 \leq n \leq m}\left|a_{n, k_{1}}-a_{n, k_{2}}\right|=\sup _{n}\left|a_{n, k_{1}}-a_{n, k_{2}}\right| & \\
& \text { uniformly in } k_{1} \text { and } k_{2}\left(1 \leq k_{1}, k_{2}<\infty\right) .
\end{aligned}
$$

Now we consider the space $b v_{0}=\left\{x \in \omega: \sum_{k=1}^{\infty}\left|x_{k}-x_{k-1}\right|<\infty\right\} \cap c_{0}$, where $x_{0}=0$; we use the convention that any term with an index less than or equal to 0 is equal to zero.

Theorem 3.5. (a) If $L \in \mathcal{B}\left(b v_{0}, c\right)$, then $L$ is given by an infinite matrix $A$ as in Proposition 2.1 (b), and the Hausdorff measure of noncompactness of $L$ satisfies the inequality

$$
\frac{1}{2} \cdot \lim _{r \rightarrow \infty}\left(\sup _{n}\left\|C_{n}^{<r>}\right\|_{\infty}\right) \leq\|L\|_{\chi} \leq \lim _{r \rightarrow \infty}\left(\sup _{n}\left\|C_{n}^{<r>}\right\|_{\infty}\right),
$$

where $C=\left(c_{n k}\right)_{n, k=1}^{\infty}$ is the matrix with $c_{n k}=\sum_{j=1}^{k} a_{n j}-\gamma_{k}$ for $n, k=1,2, \ldots$ and $\gamma_{k}=\lim _{n \rightarrow \infty} \sum_{j=1}^{k} a_{n j}$ for $k=1,2, \ldots$

(b) If $L \in \mathcal{B}\left(b v_{0}, c\right)$, then $L$ is compact if and only if

$$
\lim _{r \rightarrow \infty}\left(\sup _{n}\left\|C_{n}^{<r>}\right\|_{\infty}\right)=0 .
$$

(c) If $L \in \mathcal{B}\left(b v_{0}, \ell_{\infty}\right)$, then $L$ is compact if and only if 


$$
\begin{aligned}
\lim _{m \rightarrow \infty} \sup _{1 \leq n \leq m}\left|\sum_{j=1}^{k_{1}} a_{n j}-\sum_{j=1}^{k_{2}} a_{n j}\right| & =\sup _{n}\left|\sum_{j=1}^{k_{1}} a_{n j}-\sum_{j=1}^{k_{2}} a_{n j}\right| \\
& \text { uniformly in } k_{1} \text { and } k_{2}\left(1 \leq k_{1}, k_{2}<\infty\right) .
\end{aligned}
$$

Proof. (a) Since $b v_{0}$ is a $B K$ space with $A K$ with respect to the norm defined by $\|x\|=\sum_{k=1}^{\infty}\left|x_{k}-x_{k+1}\right|\left(x \in b v_{0}\right)$ by [19, Theorem 7.3.5 (i)], it follows from Proposition $2.1(\mathrm{~b})$ that $L \in \mathcal{B}\left(b v_{0}, c\right)$ is given by a matrix $A$. By [19, Example 8.4.2A], we have $A \in\left(b v_{0}, c\right)$ if and only if

$$
\sup _{n, k}\left|\sum_{j=1}^{k} a_{n j}\right|<\infty \text { and } \alpha_{k}=\lim _{n \rightarrow \infty} a_{n k} \text { exists for each } k \text {. }
$$

We define the matrix $\tilde{C}=\left(\tilde{c}_{n k}\right)_{n, k=1}^{\infty}$ by $\tilde{c}_{n k}=\sum_{j=1}^{k} a_{n j}$ for $n, k=1,2, \ldots$ Obviously the limits $\alpha_{k}$ exist if and only if the limits $\gamma_{k}=\lim _{n \rightarrow \infty} \tilde{c}_{n k}$ exist for each $k$. Thus we obtain by [19, Example 8.4.1A] that $A \in\left(b v_{0}, c\right)$ if and only if $\tilde{C} \in\left(\ell_{1}, c\right)$. Furthermore, Abel's summation by parts yields for each $m \in \mathbb{N}$

$$
\sum_{k=1}^{m} a_{n k} x_{k}=\sum_{k=1}^{m-1} \tilde{c}_{n k}\left(x_{k}-x_{k+1}\right)+\tilde{c}_{n m} x_{m} \text { for each fixed } n \in \mathbb{N} \text { and each } x \in b v_{0} \text {. }
$$

Since $\tilde{C}_{n} \in \ell_{\infty}$ for each $n \in \mathbb{N}$ and $x \in b v_{0} \subset c_{0}$, we obtain $A_{n} x=\tilde{C}_{n} x$ for all $n \in \mathbb{N}$ and all $x \in b v_{0}$, where $y=\left(x_{k}-x_{k+1}\right)_{k=1}^{\infty}$, hence

$$
L_{A}(x)=L_{\tilde{C}}(y) \text { for all } x \in b v_{0} \text {, where } y=\left(x_{k}-x_{k+1}\right)_{k=1}^{\infty} \text {. }
$$

Also by [18, 7.3.4], $b v_{0}$ and $\ell_{1}$ are equivalent, and also $x \in S_{b v_{0}}$ if and only if $y \in S_{\ell_{1}}$, since $\|x\|=\|y\|_{1}$. Thus we obtain from (2.6) and (3.9)

$$
\|L\|_{\chi}=\left\|L_{A}\right\|_{\chi}=\chi\left(L_{A}\left(S_{b v_{0}}\right)\right)=\chi\left(L_{\tilde{C}}\left(S_{\ell_{1}}\right)\right)=\left\|L_{\tilde{C}}\right\|_{\chi} .
$$

Finally, (3.1) with $C$ in place of $B$ and $p=1$, that is, $q=\infty$, yields (3.6).

(b) The statement is an immediate consequence of (3.6) and (2.7).

(c) As in the proof of Part (a), we obtain $A \in\left(b v_{0}, \ell_{\infty}\right)$ if and only if $\tilde{C} \in\left(\ell_{1}, \ell_{\infty}\right)$ and $L_{\tilde{C}}$ is compact by Lemma 3.4 if and only if (3.5) holds with $\tilde{c}_{n, k_{1}}$ and $\tilde{c}_{n, k_{2}}$ in place of $a_{n, k_{1}}$ and $a_{n, k_{2}}$, which is (3.8).

Remark 3.6. Similarly as in the proof of Proposition 3.3, we may apply [19, Theorem 8.3.9] with $X=b v_{0}$ and $Z=\ell_{1}, B K$ spaces with $A K, Y=Z^{\beta}=\ell_{\infty}$ and $X^{\beta}=b s$ (by [19, Theorem 7.3.5 (ii)]) to obtain $A \in\left(b v_{0}, \ell_{\infty}\right)$ if and only if $C=A^{T} \in\left(\ell_{1}, b s\right)$. Since $\ell_{1}$ and $b s$ are $B K$ spaces, we have by [10, Theorem 3.8 (a)] that $C \in\left(\ell_{1}, b s\right)$ if and only if $D \in\left(\ell_{1}, \ell_{\infty}\right)$, where $D$ is the matrix with the rows $D_{n}=\sum_{j=1}^{n} C_{j}$ for $n=1,2, \ldots$ So $L_{D}$ is compact by Lemma 3.4 if and only if (3.5) holds with $d_{n, k_{1}}$ and $d_{n, k_{2}}$ in place of $a_{n, k_{1}}$ and $a_{n, k_{2}}$, that is,

$$
\begin{aligned}
\lim _{m \rightarrow \infty} \sup _{1 \leq n \leq m}\left|\sum_{j=1}^{n} a_{k_{1} j}-\sum_{j=1}^{n} a_{k_{2} j}\right|=\sup _{n}\left|\sum_{j=1}^{n} a_{k_{1} j}-\sum_{j=1}^{n} a_{k_{2} j}\right| \\
\text { uniformly in } k_{1} \text { and } k_{2}\left(1 \leq k_{1}, k_{2}<\infty\right) .
\end{aligned}
$$


Next we consider some spaces of difference sequences. Let $\Delta=\left(\delta_{n k}\right)_{n, k=1}^{\infty}$ be the matrix of the first order difference, that is, $\delta_{n n}=1, \delta_{n, n-1}=-1$ and $\delta_{n k}=0$ otherwise. Its inverse is the matrix $\Sigma=\left(\sigma_{n k}\right)_{n, k=1}^{\infty}$ with $\sigma_{n k}=1$ for $1 \leq k \leq n$ and $\sigma_{n k}=0$ for $k>n(n=1,2, \ldots)$. If $X$ is any of the spaces $c_{0}, c$ or $\ell_{\infty}$, then we write $X(\Delta)=X_{\Delta}$. We need the following results.

Proposition 3.7. Let $R$ denote the transpose of the matrix $\Sigma$ and $\mathbf{n}=(n)_{n=1}^{\infty}$. Then we have

(a) $a \in\left(c_{0}(\Delta)\right)^{\beta}$ if and only if

$$
R a \in \ell_{1} \text { and } R a \in \mathbf{n}^{-1} * \ell_{\infty} ;
$$

(b) $a \in\left(\ell_{\infty}(\Delta)\right)^{\beta}$ if and only if

$$
R a \in \ell_{1} \text { and } R a \in \mathbf{n}^{-1} * c_{0} ;
$$

furthermore $(c(\Delta))^{\beta}=\left(\ell_{\infty}(\Delta)\right)^{\beta}$.

(c) If $a \in(X(\Delta))^{\beta}$, then we have

$$
\sum_{k=1}^{\infty} a_{k} x_{k}=\sum_{k=1}^{\infty}\left(R_{k} a\right)\left(\Delta_{k} x\right) \text { for all } x \in X .
$$

Proof. (a), (b) If $X=c_{0}$ or $X=\ell_{\infty}$, we apply [11, Theorem 3.2 and Remark 3.3 (a)] with $T=\Delta$ and $S=\Sigma$ to obtain $a \in(X(\Delta))^{\beta}$ if and only if $R a \in X^{\beta}=\ell_{1}$, which is the first condition in (3.10) and (3.11), and $W \in\left(X, c_{0}\right)$, where $W$ is the matrix with the rows $W_{m}=R_{m} a \cdot e^{[m]}$ for $m=1,2, \ldots$ Now we have by [19, Example 8.4.5A] $W \in\left(c_{0}, c_{0}\right)$ if and only if

$$
\sup _{m} \sum_{k=1}^{\infty}\left|w_{m k}\right|=\sup _{m}\left(m\left|R_{m} a\right|\right)<\infty
$$

which is the second condition in (3.10), and

$$
\lim _{m \rightarrow \infty} w_{m k}=\lim _{m \rightarrow \infty} R_{m} a=0,
$$

which is redundant. Furthermore, we have by [17, (21.1)] $W \in\left(\ell_{\infty}, c_{0}\right)$ if and only if $\lim _{m \rightarrow \infty} \sum_{k=1}^{\infty}\left|w_{m k}\right|=\lim _{m \rightarrow \infty} m\left|R_{m} a\right|=0$, which is the second condition in (3.11). Thus we have shown (a) and (b) for $c_{0}$ and $\ell_{\infty}$.

The sufficiency of the conditions in (3.11) for $a \in(c(\Delta))^{\beta}$ follows from the fact that $c(\Delta) \subset \ell_{\infty}(\Delta)$ implies $\left(\ell_{\infty}(\Delta)\right)^{\beta} \subset(c(\Delta))^{\beta}$.

Conversely, we assume $a \in(c(\Delta))^{\beta}$. Since $(c(\Delta))^{\beta} \subset\left(c_{0}(\Delta)\right)^{\beta}$, the first condition in (3.11) holds by (a). Also $\mathbf{n} \in c(\Delta)$ implies the convergence of the series $\sum_{n=1}^{\infty} k a_{k}$. This implies the second condition in (3.11) by [10, Corollary 3.16].

(c) The statement for $X=c_{0}$ or $X=\ell_{\infty}$ follows from [11, Theorem 3.2 (3.4) and Remark $3.3(\mathrm{a})]$, and also for $X=c$, since $(c(\Delta))^{\beta}=\left(\ell_{\infty}(\Delta)\right)^{\beta}$, as we have just shown.

Proposition 3.8. We have

(a) $A \in\left(c_{0}(\Delta), \ell_{\infty}\right)$ if and only if

$$
\sup _{n}\left\|\hat{A}_{n}\right\|_{1}<\infty, \text { where } \hat{A}_{n}=R A_{n}=\left(\sum_{j=k}^{\infty} a_{n j}\right)_{k=1}^{\infty}
$$


and

$$
\sup _{m}\left|m R_{m} A_{n}\right|<\infty \text { for each } n ;
$$

(b) $A \in\left(\ell_{\infty}(\Delta), \ell_{\infty}\right)$ if and only if (3.13) holds and

$$
\lim _{m \rightarrow \infty} m R_{m} A_{n}=0 \text { for each } n
$$

furthermore $\left(c(\Delta), \ell_{\infty}\right)=\left(\ell_{\infty}(\Delta), \ell_{\infty}\right)$.

(c) If $A \in\left(X(\Delta), \ell_{\infty}\right)$, then we have

$$
A x=\hat{A}(\Delta x) \text { for all } x \in X(\Delta)
$$

and

$$
\left\|L_{A}\right\|=\left\|L_{\hat{A}}\right\|
$$

where $\hat{A} \in\left(X, \ell_{\infty}\right)$.

Proof. All the statements for $X=c_{0}$ or $X=\ell_{\infty}$ are an immediate consequence of [11, Theorem 3.4, Remark 3.5 (a), Theorem 3.6] and the fact that $\left(\ell_{\infty}, \ell_{\infty}\right)=$ $\left(c_{0}, \ell_{\infty}\right)$.

The inclusion $\left(\ell_{\infty}(\Delta), \ell_{\infty}\right) \subset\left(c(\Delta), \ell_{\infty}\right)$ is clear.

Conversely we assume $A \in\left(c(\Delta), \ell_{\infty}\right)$. This clearly implies $A \in\left(c_{0}(\Delta), \ell_{\infty}\right)$ and so the condition in (3.13) holds. Also $A \in\left(c(\Delta), \ell_{\infty}\right)$ implies $A_{n} \in(c(\Delta))^{\beta}$ for all $n$, and since $(c(\Delta))^{\beta}=\left(\ell_{\infty}(\Delta)\right)^{\beta}$ by Proposition $3.7(\mathrm{~b})$, the condition in (3.15) follows from the second condition in (3.11). Now the conditions in (3.13) and $(3.15)$ imply $A \in\left(\ell_{\infty}(\Delta), \ell_{\infty}\right)$, and so we have also shown $\left(c(\Delta), \ell_{\infty}\right) \subset$ $\left(\ell_{\infty}(\Delta), \ell_{\infty}\right)$.

The identity in (3.17) is a consequence of that in (3.16) and the fact that the $B K$ norms of the spaces $c_{0}(\Delta), c(\Delta)$ are the same; (3.17) for $c_{0}(\Delta)$ follows from [10, Theorem 3.6]; for $\ell_{\infty}(\Delta)$ from [10, Remark 3.5] (which gives (3.16) for $\ell_{\infty}(\Delta)$ ) and the definition of the norms of the operators $L_{A}$ and $L_{\hat{A}}$. Finally, clearly (3.12) implies (3.16) for $c(\Delta)$ and (3.17) follows from the definition of the norms of the operators $L_{A}$ and $L_{\hat{A}}$. Indeed, we have the relations

$$
\begin{aligned}
\left\|L_{A}\right\|=\sup _{z \in B_{c}(\Delta)}\left\|L_{A} z\right\|_{\ell_{\infty}} & =\sup _{z \in B_{c(\Delta)}}\left\|L_{\hat{A}}(\Delta z)\right\|_{\ell_{\infty}}= \\
& =\sup _{\Delta z \in B_{c}}\left\|L_{\hat{A}}(\Delta z)\right\|_{\ell_{\infty}}=\sup _{y \in B_{c}}\left\|L_{\hat{A}} y\right\|_{\ell_{\infty}}=\left\|L_{\hat{A}}\right\|
\end{aligned}
$$

and the proof is completed.

Theorem 3.9. Let $X$ denote any of the spaces $c_{0}$, c and $\ell_{\infty}$. If $A \in\left(X(\Delta), \ell_{\infty}\right)$ then $L_{A}$ is compact if and only if

$$
\lim _{r \rightarrow \infty}\left(\sup _{n}\left\|\hat{A}_{n}^{>r<}\right\|_{1}\right)=\lim _{r \rightarrow \infty}\left(\sup _{n} \sum_{k=r}^{\infty}\left|\sum_{j=k}^{\infty} a_{n j}\right|\right)=0 .
$$

Proof. Let $A \in\left(X(\Delta), \ell_{\infty}\right)$. Then we have $\hat{A} \in\left(X, \ell_{\infty}\right)$ and $A x=\hat{A}(\Delta x)$ for all $x \in X(\Delta)$ by Proposition 3.8 (c) and (3.16). Since $\left(X, \ell_{\infty}\right)=\left(c_{0}, \ell_{\infty}\right)$ by [19, Example 8.4.5A], we obtain $\hat{A} \in\left(X, \ell_{\infty}\right)$ if and only if $\hat{B}=\hat{A}^{T} \in\left(\ell_{1}, \ell_{1}\right)$ by [19, 
Theorem 8.3.9], and by [10, Theorem 2.28], $L_{\hat{B}} \in \mathcal{B}\left(\ell_{1}, \ell_{1}\right)$ is compact if and only if

$$
\begin{aligned}
\lim _{r \rightarrow \infty}\left(\sup _{k} \sum_{n=r}^{\infty}\left|\hat{b}_{n k}\right|\right) & =\lim _{r \rightarrow \infty}\left(\sup _{k} \sum_{n=r}^{\infty}\left|\hat{a}_{k n}\right|\right) \\
& =\lim _{r \rightarrow \infty}\left(\sup _{k}\left\|\hat{A}_{k}^{>r<}\right\|_{1}\right)=\lim _{r \rightarrow \infty}\left(\sup _{n}\left\|\hat{A}_{n}^{>r<}\right\|_{1}\right) \\
& =\lim _{r \rightarrow \infty}\left(\sup _{n} \sum_{k=r}^{\infty}\left|\sum_{j=k}^{\infty} a_{n j}\right|\right)=0 .
\end{aligned}
$$

Finally, since $L_{\hat{A}}$ is compact if and only if $L_{\hat{B}}$ is compact by [16, Theorem 3] and $L_{A}$ is compact if and only if $L_{\hat{A}}$ is compact by (3.16), we have proved the statement of the theorem.

Finally, we consider the spaces $w_{\infty}$ and $[c]_{\infty}$ related to the concepts of strong $C_{1}$ boundedness and strong boundedness, studied by Maddox [7] and Kuttner and Thorpe [6], respectively, where

$$
w_{\infty}=\left\{x \in \omega: \sup _{n}\left(\frac{1}{n} \sum_{k=1}^{n}\left|x_{k}\right|\right)<\infty\right\} \text { and }[c]_{\infty}=\mathbf{n}^{-1} *\left(w_{\infty}\right)_{\Delta} .
$$

It is well known that $w_{\infty}$ is a $B K$ space with the norm $\|\cdot\|_{w_{\infty}}$ defined by

$$
\|x\|_{w_{\infty}}=\sup _{\nu} \frac{1}{2^{\nu}} \sum_{k=2^{\nu}}^{2^{\nu+1}-1}\left|x_{k}\right| \text { for all } x \in w_{\infty} .
$$

We also consider the space $v_{\infty}=\left(w_{\infty}\right)_{\Delta}$. For $\nu=0,1, \ldots$, we write $\sum_{\nu}$ and $\max _{\nu}$ for the sum and maximum taken over all indices $k$ with $2^{\nu} \leq k \leq 2^{\nu+1}-1$, and

$$
\mathcal{W}=\left\{a \in \omega:\|a\|_{\mathcal{W}}=\sum_{\nu=0}^{\infty} 2^{\nu} \max _{\nu}\left|a_{k}\right|<\infty\right\} .
$$

Theorem 3.10. Writing $\sum_{\mu}$ for the sum taken over all indices $n$ with $2^{\mu} \leq n \leq$ $2^{\mu+1}-1$, we have

(a) $A \in\left(\ell_{1}, w_{\infty}\right)$ if and only if

$$
\sup _{\mu, k}\left(\frac{1}{2^{\mu}} \sum_{\mu}\left|a_{n k}\right|\right)<\infty
$$

(b) $A \in\left(\ell_{1}, v_{\infty}\right)$ if and only if

$$
\sup _{\mu, k}\left(\frac{1}{2^{\mu}} \sum_{\mu}\left|a_{n k}-a_{n-1, k}\right|\right)<\infty ;
$$

(c) $A \in\left(\ell_{1},[c]_{\infty}\right)$ if and only if

$$
\sup _{\mu, k}\left(\frac{1}{2^{\mu}} \sum_{\mu}\left|n a_{n k}-(n-1) a_{n-1, k}\right|\right)<\infty
$$


Proof. (a) We apply [19, Theorem 8.3.9] with $X=\ell_{1}$ and $Z=\mathcal{W}$, a $B K$ space with $A K$ with respect to $\|\cdot\|_{\mathcal{W}}$ by [4, Proposition $\left.2.4(\mathrm{~b})\right]$, and $Y=Z^{\beta}=w_{\infty}$ by [3, Proposition $1.2(\mathrm{c})]$ to obtain $A \in\left(\ell_{1}, w_{\infty}\right)$ if and only if $B=A^{T} \in\left(\mathcal{W}, \ell_{\infty}\right)$. Since, by [4, Proposition 2.4 (c)],

$$
\|a\|_{\mathcal{W}}^{*}=\|a\|_{w_{\infty}}=\sup _{\nu}\left(\frac{1}{2^{\nu}} \sum_{\nu}\left|a_{k}\right|\right) \text { on } \mathcal{W}^{\beta}
$$

we obtain by [10, Theorem $1.23(\mathrm{~b})] B \in\left(\mathcal{W}, \ell_{\infty}\right)$ if and only if

$$
\sup _{n}\left\|B_{n}\right\|_{\mathcal{W}}^{*}=\sup _{n, \nu}\left(\frac{1}{2^{\nu}} \sum_{\nu}\left|b_{n k}\right|\right)=\sup _{n, \nu}\left(\frac{1}{2^{\nu}} \sum_{\nu}\left|a_{k n}\right|\right)=\sup _{\mu, k}\left(\frac{1}{2^{\mu}} \sum_{\mu}\left|a_{n k}\right|\right)<\infty .
$$

(b) We have by [10, Theorem $3.8(\mathrm{a})] A \in\left(\ell_{1}, v_{\infty}\right)$ if and only if $C=\left(c_{n k}\right)_{n, k=1}^{\infty}=$ $\Delta \cdot A \in\left(\ell_{1}, w_{\infty}\right)$. Since $c_{n k}=a_{n k}-a_{n-1, k}$ for all $n$ and $k,(3.21)$ is an immediate consequence of (3.20).

(c) We have by [10, Theorem 3.8 (a)] $A \in\left(\ell_{1},[c]_{\infty}\right)$ if and only in $D(\mathbf{n}) \cdot A \in$ $\left(\ell_{1}, v_{\infty}\right)$ where $D(\mathbf{n})$ is the diagonal matrix with the sequence $\mathbf{n}$ on its diagonal. Now (3.22) is an immediate consequence of (3.21).

Finally we give the characterizations for compact operators $L \in B\left(\ell_{1}, Y\right)$, where $Y$ is any of the spaces $w_{\infty}, v_{\infty}$ or $[c]_{\infty}$.

Theorem 3.11. Writing $\sum_{\mu_{1}}$ and $\sum_{\mu_{2}}$ for the sums taken over all indices $n$ with $2^{\mu_{1}} \leq n \leq 2^{\mu_{1}+1}-1$ and $2^{\mu_{2}} \leq n \leq 2^{\mu_{2}+1}-1$, we have:

(a) If $L \in \mathcal{B}\left(\ell_{1}, w_{\infty}\right)$, then $L$ is compact if and only if

$$
\begin{array}{r}
\lim _{j \rightarrow \infty}\left(\sup _{1 \leq k \leq j}\left|\frac{1}{2^{\mu_{1}}} \sum_{\mu_{1}} a_{n k}-\frac{1}{2^{\mu_{2}}} \sum_{\mu_{2}} a_{n k}\right|\right)=\sup _{k}\left|\frac{1}{2^{\mu_{1}}} \sum_{\mu_{1}} a_{n k}-\frac{1}{2^{\mu_{2}}} \sum_{\mu_{2}} a_{n k}\right| \\
\text { uniformly in } \mu_{1} \text { and } \mu_{2}\left(0 \leq \mu_{1}, \mu_{2}<\infty\right) .
\end{array}
$$

(b) If $L \in \mathcal{B}\left(\ell_{1}, v_{\infty}\right)$, then $L$ is compact if and only if

$$
\begin{array}{r}
\lim _{j \rightarrow \infty}\left(\sup _{1 \leq k \leq j}\left|\frac{1}{2^{\mu_{1}}}\left(a_{2^{\mu_{1}+1}-1, k}-a_{2^{\mu_{1}}-1, k}\right)-\frac{1}{2^{\mu_{2}}}\left(a_{2^{\mu_{2}+1}-1, k}-a_{2^{\mu_{2}-1, k}}\right)\right|\right)= \\
\sup _{k}\left|\frac{1}{2^{\mu_{1}}}\left(a_{2^{\mu_{1}+1}-1, k}-a_{2^{\mu_{1}}-1, k}\right)-\frac{1}{2^{\mu_{2}}}\left(a_{2^{\mu_{2}+1}-1, k}-a_{2^{\mu_{2}-1, k}}\right)\right| \\
\text { uniformly in } \mu_{1} \text { and } \mu_{2}\left(0 \leq \mu_{1}, \mu_{2}<\infty\right) .
\end{array}
$$

(c) If $L \in \mathcal{B}\left(\ell_{1},[c]_{\infty}\right)$, then $L$ is compact if and only if

$$
\begin{gathered}
\lim _{j \rightarrow \infty}\left(\sup _{1 \leq k \leq j} \mid \frac{1}{2^{\mu_{1}}}\left(\left(2^{\mu_{1}+1}-1\right) a_{2^{\mu_{1}+1}-1, k}-\left(2^{\mu_{1}}-1\right) a_{2^{\mu_{1}}-1, k}\right)-\right. \\
\left.-\frac{1}{2^{\mu_{2}}}\left(\left(2^{\mu_{2}+1}-1\right) a_{2^{\mu_{2}+1}-1, k}-\left(2^{\mu_{2}}-1\right) a_{2^{\mu_{2}-1, k}}\right) \mid\right)= \\
\sup _{k} \mid \frac{1}{2^{\mu_{1}}}\left(\left(2^{\mu_{1}+1}-1\right) a_{2^{\mu_{1}+1}-1, k}-\left(2^{\mu_{1}}-1\right) a_{2^{\mu_{1}}-1, k}\right)- \\
\quad-\frac{1}{2^{\mu_{2}}}\left(\left(2^{\mu_{2}+1}-1\right) a_{2^{\mu_{2}+1}-1, k}-\left(2^{\mu_{2}}-1\right) a_{2^{\mu_{2}-1, k}}\right) \mid
\end{gathered}
$$




$$
\text { uniformly in } \mu_{1} \text { and } \mu_{2}\left(0 \leq \mu_{1}, \mu_{2}<\infty\right) \text {. }
$$

Proof. (a) This is the case $p=1$ in [3, Corollary $\left.3.147 .(7.1)^{+}\right]$.

(b) By Theorem 3.10 (a) and (b), we have $A \in\left(\ell_{1}, v_{\infty}\right)$ if and only if $C=$ $\Delta \cdot A \in\left(\ell_{1}, w_{\infty}\right)$ and so the condition in (3.24) is an immediate consequence of that in $(3.23)$.

(c) By Theorem 3.10 (b) and (c), we have $A \in\left(\ell_{1},[c]_{\infty}\right)$ if and only if $D(\mathbf{n}) \cdot A \in$ $\left(\ell_{1}, v_{\infty}\right)$ and so the condition in (3.25) is an immediate consequence of that in (3.24).

Acknowledgement. Research of the Djolović and Malkowsky supported by the research projects 174007 and 174025, respectively, of the Serbian Ministry of Science, Technology and Environmental Development. Research of Malkowsky also supported by the research project TUBITAK Project No. 114F104.

\section{REFERENCES}

1. E.A. Alekhno, The lower Weyl spectrum of a positive operator, Integral Equations Operator Theory 67 (2010), no. 3, 301-326.

2. I. Djolović and E. Malkowsky, A note on compact operators on matrix domains, J. Math. Anal. Appl. 340 (2008), 291-303.

3. I. Djolović and E. Malkowsky, Compact operators into the spaces of strongly $C_{1}$ summable and bounded sequences, Nonlinear Anal. 74 (2011), 3736-3750.

4. I. Djolović and E. Malkowsky, Banach algebras of matrix transformations between some sequence spaces related to $\Lambda$-strong convergence and boundedness, Appl. Math. Comp. 219 (2013), 8779-8789.

5. A.M. Jarrah and E. Malkowsky, Ordinary, absolute and strong summability, Filomat 17 (2003), 59-78.

6. B. Kuttner and B. Thorpe, Strong convergence, J. Reine Angew. Math. 311/312 (1979), $42-55$.

7. I.J. Maddox, On Kuttner's theorem, J. London Math. Soc. 43 (1968) 285-290.

8. B. de Malafosse, E. Malkowsky and V. Rakočević, Measure of noncompactness of operators and matrices on the spaces $c$ and $c_{0}$, Int. J. Math. Math. Sci. (2006), Issue 1, Article ID 46930, 5 pages.

9. B de Malafosse and V. Rakočević, Application of measure of noncompactness in operators on the spaces $s_{\alpha}, s_{\alpha}^{0}, s_{\alpha}^{(c)}, \ell_{\alpha}^{p}$, J. Math. Anal. Appl. 323 (2006), no. 1, 131-145.

10. E. Malkowsky and V. Rakočević, An Introduction into the Theory of Sequence Spaces and Measures of Noncompactness, Zb. Rad. (Beogr.) 9(17) (2000), 143-234.

11. E. Malkowsky and V. Rakočević, On matrix domains of triangles, Appl. Math. Comp. 189 (2007) 1146-1163.

12. M. Mursaleen and S.A. Mohiuddine, Applications of measures of noncompactness to infinite systems of differential equations in $\ell_{p}$ spaces, Nonlinear Anal. 75 (2012), 2111-2115.

13. M. Mursaleen and A.M. Noman, Compactness by the Hausforff measure of noncompactness, Nonlinear Anal. 73 (2010) 2541-2557.

14. M. Mursaleen and A.M. Noman, Applications of the Hausdorff measure of noncompactness in some sequence spaces of weighted means, Comput. Math. Appl. 60 (2010) 1245-1258.

15. M. Mursaleen and V. Karakaya, H. Polat and N. Simşek, Measure of noncompactness of matrix operators in some difference sequences of weighted means, Comput. Math. Appl. 62 (2011) 814-820.

16. W.L.C. Sargent, On compact matrix transformations between sectionally bounded BKspaces, J. London Math. Soc. 41 (1966), 79-87. 
17. M. Stieglitz and H. Tietz, Matrixtransformationen in Folgenräumen, Math. Z. 154 (1977), $1-16$.

18. J.M. Ayerbee Toledano, T. Dominguez Benavides and G. Lopez Acedo, Measures of Noncompactness in Metric Fixed Point Theory, Operator Theory, Advances and Applications, Vol 99, Birkhäuser, Basel, Boston, Berlin, 1997.

19. A. Wilansky, Summability Through Functional Analysis, North-Holland Mathematics Studies 85, Amsterdam, 1984.

${ }^{1}$ Department of Mathematics, Faculty of Science, Fatih University, BÜYÜKÇEKMECE 34500, Istanbul, TuRKey;

C/o Državni Univerzitet, u Novom Pazaru, Vuka Karadžića bB, 36300 Novi Pazar, SERBIA.

E-mail address: Eberhard.Malkowsky@math.uni-giessen.de; ema@Bankerinter.net

${ }^{2}$ Technical Faculty, University of Belgrade, VJ 12, 19210 Bor, Serbia.

E-mail address: zucko@open.telekom.rs

${ }^{3}$ Faculty of Civil Engineering and Architecture, University of Niš, AleksanDra Medvedova 14, 18000 Niš, SERBia.

E-mail address: katarina.petkovic@gaf.ni.ac.rs 